\title{
Normas de Desempenho em Compreensão de Leitura Textual para Crianças de 10 Ano a 6ª Série
}

\author{
Helena Vellinho Corso \\ Luciane Rosa Piccolo \\ Camila Schorr Miná \\ Jerusa Fumagalli Salles \\ Universidade Federal do Rio Grande do Sul \\ Porto Alegre, RS, Brasil
}

\begin{abstract}
RESUMO
Este estudo apresenta as normas de desempenho para um instrumento, composto de duas tarefas diferentes, que avalia a compreensão leitora de textos narrativos. As tarefas de reconto (recordação livre) e questionário a partir da história "A Coisa" permitem avaliar crianças de 1 ㅇ a $3^{\circ}$ anos, enquanto as mesmas tarefas a partir da história "O Coelho e o Cachorro" avaliam crianças que cursam o $4^{\circ}$ ano até a $6^{a}$ série. Participaram do estudo 176 crianças $(51,7 \%$ meninas) com idades entre sete e 12 anos, de primeiro ano à sexta série do Ensino Fundamental, de escolas públicas e privadas de Porto Alegre-RS. Os escores normativos para crianças de $1^{\underline{O}}$ ano a $6^{\underline{a}}$ série do Ensino Fundamental foram divididos por anos completos de escolarização e tipo de escola. Foram realizadas análises descritivas (média e desvio padrão) e comparação dos escores nas tarefas de compreensão textual segundo os anos completos de estudo formal e tipo de escola (públicas e privadas) das crianças (Mann-Whitney e Kruskal-Wallis). As crianças de anos escolares mais avançados apresentaram desempenho superior àquelas de séries iniciais. Estudantes de escolas privadas tiveram desempenho superior àqueles de escolas públicas. $\mathrm{O}$ instrumento mostra-se útil para diferenciar desempenhos para diferentes escolaridades e tipos de escolas e é de fácil aplicação. O instrumento pode ser utilizado por clínicos, pesquisadores e profissionais do meio escolar.
\end{abstract}

Palavras-chave: Compreensão leitora; Linguagem escrita; Avaliação da linguagem; Leitura; Crianças.

\begin{abstract}
Developmental Standards in a Reading Comprehension Task for Elementary School Children

This study presents the performance standards for an assessment tool, constituted by two different tasks, elaborated to evaluate reading comprehension of narrative texts. Retelling and a questionnaire based on the story "The Thing" permitted the evaluation of children from the 1st to 3rd grades, whereas the same tasks based on the story "The Rabbit and the Dog" assessed children from the 4 th to 6 th grade. The study included 176 children $(51.7 \%$ girls) between seven and 12 years old, from the first to sixth grade of elementary school, from public and private schools in Porto Alegre-RS. Normative scores for children from the 1st to 6th grades were divided by years of education and type of school. Descriptive statistics (mean and standard deviation) and comparison of the scores on the reading comprehension tasks were carried out using years of education and type of school (public and private) of children (Mann-Whitney and Kruskal-Wallis). Compared to younger children from initial grades, older children from advanced grades showed a better performance in reading comprehension. Students from private schools performed better when compared to students from public schools. The assessment tool seems to be useful to distinguish performances of children from different years of education and type of schools and it is easily applied. Clinicians, researchers and professionals in the school environment can use the instrument.
\end{abstract}

Keywords: Reading comprehension; Written language; Language assessment; Reading; Children.

\section{RESUMEN}

\section{Normas de Desempeño en Tareas de Comprensión de Lectura para Niños en Escuela Primaria}

Este estudio presenta normas de desempeño de un instrumento compuesto de dos tareas diferentes, que evalúa la comprensión de textos narrativos. La tarea de volver a contar (recuerdo libre) y el cuestionario de la historia "La Cosa" permite evaluar los niños del primero al tercero grados, mientras que las mismas tareas desde la historia "El Conejo y el Perro" evalúan a los niños entre cuarto e sexto grados. El estudio incluyó a 176 niños (51,7\% niñas) de edades comprendidas entre los siete y los 12 años, cursando del primer año a lo sexto grado de la escuela primaria, y frequentando escuelas públicas y privadas de Porto Alegre-RS. Las puntuaciones normativas para alumnos de primero a sexto grados fueron divididos por años completos de escolaridad y tipo de escuela. Estadística descriptiva (media y desviación estándar) y la comparación de las puntuaciones en las tareas de comprensión de lectura fueran llevadas a cabo considerando los años completos de estudio formal y el tipo de escuela (pública y privada) de los niños (Mann-Whitney y Kruskal-Wallis). Niños más avanzados presentaron desempeño superior a los de grados iniciales. Estudiantes en escuelas privadas tuvieron un rendimiento superior a lo de las escuelas públicas. El instrumento ha demostrado ser útil para diferenciar el desempeño de diferentes grados y tipos de escuelas, es fácil de aplicar y, por outra parte, puede ser utilizado por clínicos, investigadores y profesionales del escuela.

Palabras clave: Comprensión de lectura; Lenguaje escrita; Evaluación de la lenguaje; Lectura; Niños. 


\section{INTRODUÇÃO}

A aquisição da leitura proficiente caracteriza um processo que se inicia pela habilidade de reconhecimento da palavra, progride na acurácia e velocidade com que esse reconhecimento é feito e culmina com a possibilidade de compreender textos que supõe, além da automaticidade na leitura de palavras, o uso de estratégias metacognitivas (Schmitt, Jiang, \& Grabe, 2011; van Kraayenoord, Beinicke, Schlagmuller, \& Schneider 2012). Acurácia e fluência no reconhecimento de palavras são aspectos centrais à leitura proficiente, influenciando a compreensão leitora propriamente dita (Cain, Oakhill, \& Bryant, 2004; Ouellette \& Beers, 2010), mas o reconhecimento de palavras não garante a compreensão do texto, já que outras habilidades, linguísticas e cognitivas, são demandadas neste processo (Kendeou, Muis, \& Fulton, 2010). É o que fica claro nos quadros de dificuldade específica em compreensão leitora, em que o significado do texto lido não é construído, apesar de a leitura de palavras encontrar-se intacta (Hulme \& Snowling, 2011; Sotelo-Dynega, Flanagan, \& Alfonso, 2011).

O desenvolvimento da compreensão leitora não corresponde apenas a causas internas, como as capacidades cognitivas e linguísticas próprias à criança, que facilitam ou dificultam a aquisição dessa habilidade. Há também causas externas, como o ensino informal, a instrução formal e as práticas de leitura (Ehri, 2010). As experiências da criança em um ambiente mais ou menos estimulador explicam em parte seu desempenho em domínios fundamentais para o desenvolvimento da leitura, como conhecimento das letras e consciência fonológica (especialmente no caso do reconhecimento da palavra) e linguagem oral (especialmente quanto à compreensão leitora) (Phillips \& Lonigan, 2010; Piccolo \& Salles, 2013).

A revisão de dois modelos psicolinguísticos importantes de compreensão leitora - o de Kintsch (Kintsch, 1988, 1998) e o de Trabasso (Trabasso, Van DenBroek, \& Suh, 1989) - permite concluir que esta habilidade caracteriza um processo muito sofisticado, no qual as unidades menores do texto vão gradualmente sendo integradas entre si e com o conhecimento do leitor. Este trabalho mental caracteriza uma construção ativa da representação final do texto, retida na mente do leitor. O resultado da leitura compreensiva não é uma cópia do texto, mas uma representação mental que resulta do estabelecimento de relações internas ao texto, da seleção de conteúdo relevante, da explicitação mental de informações não explicitadas nas passagens escritas e da integração das informações do texto às estruturas de conhecimento anteriores do leitor (Corso, Sperb, \& Salles, no prelo).

O fato de que a compreensão leitora não caracterize um, mas múltiplos processos cognitivos que provavelmente atuam de forma integrada (Kintsch \& Rawson, 2005) determina dificuldades ao processo de avaliação da habilidade. Tratando-se de um construto latente, verifica-se que diferentes medidas de compreensão podem apresentar fraca correlação e podem estar apoiadas por habilidades cognitivas diferentes (Johnston, Barnes, \& Desrochers, 2008). A utilização de tarefas diversas, com suas demandas linguísticas e cognitivas distintas, pode oferecer um entendimento mais abrangente do processo de compreensão (Brandão \& Spinillo, 1998). O instrumento normatizado neste trabalho possui duas tarefas de compreensão - reconto (evocação livre) e questionário de múltipla escolha - com base em dois textos diferentes (conforme o nível de escolaridade da criança). Considera-se que a utilização de ambas as medidas pode oferecer uma investigação mais abrangente da habilidade de compreender textos dos examinados.

Entre os instrumentos de avaliação de leitura de texto comercializados no Brasil estão "Avaliação da compreensão leitora: textos expositivos" (Saraiva, Moojen, \& Munarski, 2009), que ainda não foi normatizado, e o "PROLEC" (Cuetos, Rodrigues, \& Ruano, 1998), uma adaptação da versão original em espanhol. Este último contém dois textos narrativos e dois textos expositivos, mas são textos pequenos (o maior tem 59 palavras), seguidos de quatro questões literais (nenhuma inferencial). Mais instrumentos de avaliação da compreensão leitora são necessários para uso clínico, investigativo e escolar, utilizando textos maiores e textos narrativos, que oferecem um maior controle sobre o conhecimento prévio do leitor, assim como com critérios claros de pontuação (correção). Ao contrário do texto expositivo, que trata sempre de um domínio determinado e supõe do leitor certo grau de conhecimento prévio acerca desse domínio, o texto narrativo só exige do leitor conhecimento geral de mundo, além das habilidades linguísticas também requeridas diante do texto expositivo (Marotto, 2000).

Este estudo apresenta dados normativos de desempenho de crianças do primeiro ano à sexta série do Ensino Fundamental de escolas públicas e particulares de Porto Alegre - Rio Grande do Sul, em um instrumento de avaliação da compreensão leitora que envolve duas tarefas - o reconto (evocação livre) e o questionário - com base em dois textos narrativos, adequados de acordo com a idade/escolaridade da criança. Além disso, o estudo objetivou comparar o 
desempenho dos participantes da amostra nas tarefas de compreensão, tanto em relação aos anos completos de estudo, quanto em relação ao tipo de escola frequentada (a análise de comparação já havia sido realizada apenas junto à amostra de alunos da quarta a sexta séries. Para mais detalhes ver Corso, Sperb, \& Salles, 2013).

\section{MÉTODO}

\section{Participantes}

A amostra geral foi composta por 176 crianças (51,7\% meninas) com idades entre sete e 12 anos $(M=9,5 ; D P=1,82)$ de primeiro ano à sexta série do Ensino Fundamental, de escolas públicas $(42,6 \%)$ e privadas $(57,4 \%)$ de Porto Alegre - Rio Grande do Sul (RS).Os critérios de inclusão no estudo são ausência de: histórico de doenças neurológicas ou psiquiátricas, dificuldades auditivas ou visuais não corrigidas, indícios de dificuldades de aprendizagem (conforme indicação da professora) e repetência escolar, indicadores de alterações comportamentais e de Transtorno de Déficit de Atenção e Hiperatividade (Conners Abbreviated Teacher Rating Scale - CATRS-10, Brito, 1987). Além disso, os participantes deveriam apresentar desempenho igual ou superior ao percentil 25 no Teste de Matrizes Progressivas de Raven - Escala especial (Angelini, Alves, Custódio, Duarte, \& Duarte, 1999; Raven, Court, \& Raven, 1986).

Especificamente para o estudo normativo das tarefas de compreensão leitora da história "A Coisa", participaram 75 crianças entre sete e nove anos $(M=7,71 ; D P=0,73)$, sendo 40 meninas $(53,3 \%), 45$ estudantes de escolas particulares $(60 \%)$ e 30 estudantes de escolas públicas. Para efeitos de análises estatísticas e também buscando evitar a potencial confusão causada com a transição da 'série escolar' para o 'ano escolar', os grupos foram classificados em "anos completos de estudo". Dessa amostra, as crianças de terceiro ano são classificadas como possuindo dois anos completos de estudo, as de segundo ano possuem um ano completo de estudos, e as de primeiro ano ainda não completaram um ano de estudo formal. As tabelas do desempenho normativo também foram confeccionadas seguindo esse critério. Uma vez que não houve diferenças significativas de desempenho em compreensão de leitura textual entre os grupos frequentando o primeiro ano e com um ano de estudo completo, reuniram-se essas duas amostras de escolaridades em um só grupo $(n=43,57,3 \%)$. Os demais 32 participantes $(42,7 \%)$ tinham dois anos completos de estudo.

A amostra para a normatização da história " $O$ coelho e o cachorro" foi constituída de 101 crianças entre nove e 12 anos $(M=10,83 ; D P=1,09), 51$ me- ninas $(50,5 \%), 56$ estudantes de escolas privadas $(55,4 \%)$ e 45 de escolas públicas $(44,6 \%)$. Como não havia diferença de desempenho em compreensão de leitura textual entre os alunos de quarto ano $(n=18$; $17,8 \%)$ e quinta séries ( $n=35 ; 34,7 \%)$, essas amostras foram reunidas no mesmo grupo (53 estudantes no total, com crianças com três e quatro anos completos de estudo) e foram comparadas a $48(47,5 \%)$ crianças que cursavam a sexta série (cinco anos completos de estudo).

\section{Procedimentos gerais e instrumentos para garantia dos critérios de inclusão}

O estudo foi aprovado pelo Comitê de Ética em Pesquisa do Instituto de Psicologia (protocolo 2008/067) da UFRGS. Após contato com as escolas e assinatura dos Termos de Consentimento Livre e Esclarecido (TCLE) pelos pais/responsáveis, aplicouse coletivamente (pequenos grupos) o Teste Matrizes Progressivas Coloridas de Raven - Escala Especial (Angelini et al., 1999). Juntamente com o teste de Raven foram utilizados, para garantia dos critérios de inclusão: questionário socioeconômico (Associação Brasileira de Empresas de Pesquisa [ABEP], 2009) e de condições de saúde e escolarização (preenchidos pelos pais/responsáveis) e Escala Conners Abreviada para professores (Conners Abbreviated Teacher Rating Scale-CATRS-10, Brito, 1987).

\section{Instrumento de avaliação da compreensão leitora - caracterização, procedimentos de aplicação e pontuação}

Foram selecionados e adaptados dois textos narrativos em função de sua qualidade literária e da familiaridade do conteúdo apresentado em relação ao universo de crianças em geral (independentemente de situação socioeconômica). Os textos foram retirados de livros didáticos, considerando a adequação de séries/ anos escolares. Além disso, as duas histórias, após ajustes dos pesquisadores, favorecem a avaliação das habilidades de compreensão inferencial. Em ambos os textos foram feitas ligeiras adaptações formais, dando-se preferência por construções linguísticas mais simples e familiares às crianças de modo geral. Alguns elementos foram retirados para dar margem a criança realizar inferências no decorrer da leitura. A extensão e grau de complexidade de cada texto também foram levados em conta. "A Coisa" (Rocha, 1997), com aproximadamente 200 palavras, foi utilizado nos primeiros níveis de escolaridade da amostra (até três anos completos de estudo). "O Coelho e o Cachorro" (Prata, 2005), com 341 palavras, foi utilizado nas três últimas séries escolares. A construção do instrumento 
envolveu diferentes etapas, com a participação de juízes independentes: seleção do texto, análise de cláusulas e da estrutura macroproposicional do texto, elaboração dos questionários (Corso, Sperb, \& Salles, 2012). A correção dos recontos das histórias (evocações livres) e sua classificação nas diferentes categorias também foram feitas por juízes independentes.

Quanto aos procedimentos de aplicação, as tarefas foram realizadas em uma sessão individual com duração variável conforme a idade e escolaridade da criança (em média 10 minutos), na escola do participante, após combinação prévia com o professor e a direção da escola. Os textos, os questionários e as orientações para a aplicação dos instrumentos podem ser obtidas no site <www.ufrgs.br/neurocog $>$. Inicialmente o participante foi convidado a fazer a leitura silenciosa do texto narrativo. A criança foi instruída a ler a história atentamente e foram-lhe informados os objetivos desta leitura, ou seja, que ela seria posteriormente solicitada a recontar (evocar livremente) a história e, em seguida, a responder a questões sobre a mesma. O reconto da história é solicitado em primeiro lugar para estar o mais isento possível de interferências, já que fornecer as opções de respostas através das questões de múltipla escolha pode ser um índice de conteúdo para o reconto (Salles \& Parente, 2004).

Os recontos (evocações livres) foram gravados e posteriormente transcritos para análise e categorização. Quanto ao questionário, as questões foram lidas oralmente pelo examinador, junto com as quatro alternativas de resposta, enquanto a criança podia acompanhar a leitura visual e auditivamente. Depois de ouvir a questão e as opções de resposta, a criança devia dizer qual a alternativa que considerava correta. As dez questões de cada texto dividem-se em literais e inferenciais. Esta mesma estrutura de tarefas foi aplicada à amostra total, apenas variando o texto (e consequentemente as questões) utilizado de acordo com a série/ano escolar.

O método de construção desse instrumento está descrito em Corso, Sperb e Salles (2012). A classificação em inferências, interferências e reconstruções segue parâmetros propostos por Parente, Saboskinski, Ferreira e Nespoulous (1999). Inferências são os comentários pertinentes a respeito de fatos presentes na história original ou o relato de um fato que deve ter acontecido na história, mas que está ausente no texto original. Inferências - definidas por Oakhill e Cain (2006) como a atividade de estabelecer relações capazes de preencher mentalmente as informações deixadas implícitas no texto - são necessárias à compreensão. No sentido contrário estão as interferências, que ocorrem quando a criança modifica o significado das cláusulas por misturar no relato elementos presentes na história, mas que são independentes na história original. Também indicando falhas na compreensão está a reconstrução, que é a introdução de cláusulas que relatam fatos não presentes na história original e que não poderiam ser inferidos pelas informações contidas no texto.

\section{Análise do texto "A Coisa" e categorias de reconto}

A história foi subdividida, a partir de acordo entre três juízes, em 22 cláusulas, 11 delas fazendo parte da cadeia principal da história, todas conectadas em uma rede de sete níveis, que correspondem aos episódios ou partes da história (ver Corso \& Salles, 2009). Após a análise, os recontos podem ser classificados em cinco categorias (adaptadas de Brandão \& Spinillo, 2001) conforme o grau de compreensão, da categoria 1 que corresponde a reproduções desconectadas de frases com presença de reconstruções, até a categoria 5, que supõe uma reprodução completa da história, em que as ideias centrais e as inferências são reproduzidas de maneira articulada (para a caracterização completa das categorias ver Corso \& Salles, 2009; para exemplos de inferências, interferências e reconstruções nos recontos da história "A Coisa" ver Salles \& Parente, 2004).

\section{Análise do texto "O Coelho e o Cachorro" e categorias de reconto}

A análise - feita a partir de acordo entre três juízes - identificou 34 cláusulas. Além disso, o texto selecionado foi dividido, a partir de uma análise macroproposicional, em cinco níveis, cada um deles correspondendo a um episódio dentro da sequência típica das narrativas simples (ambientação, estabelecimento do problema, tentativa de resolução do problema, resultado/desfecho). Os recontos podem ser classificados em cinco categorias, conforme o grau de compreensão, da categoria 1 - que corresponde a relatos incompletos e incoerentes, com presença de interferências e reconstruções -, até a categoria 5-que compreende recontos completos e coerentes, muitas vezes com a presença de inferências. A caracterização completa de cada categoria, juntamente com exemplos de recontos a ela correspondentes, pode ser encontrado em Corso, Sperb e Salles (2012).

Quanto ao questionário sobre as histórias "A Coisa" e "O Coelho e o Cachorro" (publicados respectivamente em Salles \& Parente, 2004; e Corso, Sperb, \& Salles, 2012), a pontuação corresponde ao número de questões corretas (escore máximo $=10$ ), sendo que são pontuadas separadamente as questões literais (escore máximo $=5$ ) e as questões inferenciais (escore máximo $=5)$. Neste estudo de normatização, os 
resultados desta tarefa são apresentados em termos de médias de questões respondidas corretamente.

\section{Análise de dados}

Foram realizadas análises descritivas (média e desvio padrão) e comparação dos escores nas tarefas de compreensão textual segundo os anos completos de estudo formal e tipo de escola (públicas e privadas) das crianças da amostra, utilizando-se os testes Mann-Whitney e Kruskal-Wallis. Para as análises consideraram-se os escores do questionário (total de questões, questões literais e inferenciais), a categoria de reconto e a porcentagem de cláusulas da cadeia principal da história evocadas pela criança.

Em função dos dados de desempenho das crianças nas tarefas de compreensão leitora não seguir uma distribuição normal, no cálculo dos valores normativos foram utilizados percentis (porcentagem de escores/ casos que atingiram determinado escore no teste). Relacionando estes aos escores padronizados $(z)$, o percentil 15 ou 16 (valores de escore $z$ de -1,02 a -1,05) pode ser comparado a -1 desvio padrão (sugestivo de alerta para déficit); o percentil 7 (valores de escore $\mathrm{z}$ de $-1,44$ a -1,51) equivale a $-1,5$ desvio padrão (sugestivo de déficit moderado a severo) e o percentil 2 a 2,5 é equivalente a -2 desvios padrão. Escores $z$ entre -1,96 a $-2,19$ são sugestivos de déficit de gravidade importante (Strauss, Sherman, Spreen, \& Slick, 2006). Os dados foram analisados através do programa SPSS, versão 20.

\section{RESULTADOS}

As normas para ambos os textos (e respectivas amostras) são apresentadas a seguir conforme tipo de escola e anos completos de estudo formal. Não houve diferenças de desempenho nas tarefas de compreensão leitora entre meninos e meninas em nenhum dos textos, indicando que as normas servirão para crianças de ambos os sexos. Não são apresentados dados normativos para número de inferências, interferências e reconstruções. Apesar de serem dados menos objetivos que os demais no momento da pontuação, não deixam de ser levados em conta na categorização dos recontos. As frequências de alunos nas cinco categorias de classificação dos recontos de ambas histórias são apresentadas na Tabela 1 e podem ser utilizadas como norteadoras para verificar se o desempenho da criança está de acordo com a maioria das crianças de mesma escolaridade e tipo de escola.

\section{História "A Coisa" ( $1^{\circ}$ a $3^{\circ}$ ano do Ensino Fundamental)}

Os dados normativos para a tarefa de compreensão leitora - texto "A Coisa" - são apresentados na Tabela 2, por anos completos de estudo e por tipo de escola. Foi verificado efeito teto no questionário $(40 \%$ da amostra pontuou em nível máximo no questionário total; $53,3 \%$ atingiu o máximo de acertos nas questões literais, e 54,7\% nas inferenciais), mas não na tarefa de reconto da história (12\% alcançaram a categoria máxima).

Apesar de uma porcentagem importante da amostra ter escore máximo (40\% obtiveram 10 acertos no questionário total; $54,7 \%$ obtiveram 5 acertos no questionário literal; $54,7 \%$ acertaram as 5 questões inferenciais), foi possível verificar uma diferença estatisticamente significativa entre os grupos em função do tempo de escolarização nesta tarefa. Assim, os alunos com dois anos completos de estudo (frequentando $3^{\circ}$ ano) tiveram desempenho superior ao das crianças com menos anos de escolarização nos escores do questionário total $(U=494,5 ; z=-2,16$; $p<0,05)$. Analisando-se os dois questionários que compõem o escore total, verifica-se no teste de MannWhitney que esta diferença recaiu sobre as questões literais apenas $(U=454 ; z=-2,74 ; p<0,01)$.

TABELA 1

Percentual de crianças em cada uma das categorias de desempenho em compreensão de leitura (reconto) das histórias "A Coisa" e "O Coelho e o Cachorro", por tipo de escola e por anos completos de estudo

\begin{tabular}{lcccccccc}
\hline \multicolumn{1}{c}{ História } & \multicolumn{4}{c}{ "A Coisa" } & \multicolumn{3}{c}{ "O coelho e o cachorro" } \\
\hline Escolaridade & \multicolumn{2}{c}{ Até 1 ano } & \multicolumn{2}{c}{ 2 anos } & \multicolumn{2}{c}{3 e 4 anos } & \multicolumn{2}{c}{5 anos } \\
\hline Tipo de Escola & Pub & Priv & Pub & Priv & Pub & Priv & Pub & Priv \\
\hline Categoria I & 29,4 & 7,7 & 15,4 & 5,3 & 38,9 & 5,7 & 18,5 & 9,5 \\
Categoria II & 35,3 & 19,2 & 23,1 & 15,8 & 16,7 & 11,4 & 11,1 & 4,8 \\
Categoria III & 11,8 & 30,8 & 30,8 & 36,8 & 27,8 & 11,4 & 11,1 & 4,8 \\
Categoria IV & 17,6 & 30,8 & 15,4 & 26,3 & 11,1 & 37,1 & 37,0 & 38,1 \\
Categoria V & 5,9 & 11,5 & 15,4 & 15,8 & 5,6 & 34,3 & 22,2 & 42,9 \\
\hline
\end{tabular}

Legenda: Pub = Escola Pública $;$ Priv = Escola Privada. 
TABELA 2

Desempenho e percentis nas tarefas de compreensão de leitura da história "A Coisa", por tipo de escola e anos completos de estudo

\begin{tabular}{|c|c|c|c|c|c|c|c|c|c|c|}
\hline \multirow[b]{2}{*}{ Tipo de Escola } & \multicolumn{2}{|c|}{$\begin{array}{l}\text { Questionário } \\
\text { Total } \\
\text { (Máximo =10) }\end{array}$} & \multicolumn{2}{|c|}{$\begin{array}{l}\text { Questionário } \\
\text { Literal } \\
(\text { Máximo }=5)\end{array}$} & \multicolumn{2}{|c|}{$\begin{array}{l}\text { Questionário } \\
\text { Inferencial } \\
\text { (Máximo =5) }\end{array}$} & \multicolumn{2}{|c|}{$\begin{array}{c}\text { Total Cláusulas } \\
\text { Recontadas } \\
\text { (Máximo=22) }\end{array}$} & \multicolumn{2}{|c|}{$\begin{array}{c}\text { Porcentagem } \\
\text { Macroestrutura }\end{array}$} \\
\hline & $P u b$ & Priv & $P u b$ & Priv & $P u b$ & Priv & Pub & Priv & $P u b$ & Priv \\
\hline \multicolumn{11}{|c|}{ Até 1 ano de escolaridade $(n=43)$} \\
\hline \multicolumn{11}{|l|}{ Percentil } \\
\hline 2,5 & 3,0 & 6,0 & 1,0 & 2,0 & 1,0 & 3,0 & 0,0 & 0,0 & 0,0 & 0,0 \\
\hline 7 & 3,3 & 6,0 & 1,3 & 2,9 & 1,3 & 3,0 & 0,0 & 2,7 & 0,0 & 8,0 \\
\hline 10 & 3,8 & 6,7 & 1,8 & 3,0 & 1,8 & 3,0 & 0,0 & 3,7 & 0,0 & 15,3 \\
\hline 16 & 4,0 & 7,0 & 2,0 & 3,0 & 2,0 & 3,3 & 0,0 & 4,6 & 0,0 & 20,9 \\
\hline 20 & 5,2 & 7,0 & 2,0 & 3,0 & 2,6 & 4,0 & 0,6 & 6,0 & 5,4 & 31,0 \\
\hline 25 & 6,0 & 7,0 & 2,5 & 3,0 & 3,0 & 4,0 & 1,5 & 6,8 & 9,0 & 43,0 \\
\hline 30 & 6,4 & 8,0 & 3,0 & 3,0 & 3,0 & 4,0 & 2,8 & 7,1 & 12,6 & 45,0 \\
\hline 40 & 7,0 & 8,0 & 4,0 & 4,0 & 4,0 & 4,8 & 5,2 & 8,0 & 27,0 & 53,0 \\
\hline 50 & 8,0 & 9,0 & 4,0 & 5,0 & 4,0 & 5,0 & 6,0 & 9,5 & 36,0 & 55,0 \\
\hline 60 & 8,0 & 10,0 & 4,0 & 5,0 & 4,0 & 5,0 & 6,8 & 10,0 & 43,2 & 64,0 \\
\hline 70 & 8,6 & 10,0 & 4,0 & 5,0 & 5,0 & 5,0 & 8,0 & 11,9 & 51,0 & 64,0 \\
\hline 75 & 9,0 & 10,0 & 4,0 & 5,0 & 5,0 & 5,0 & 9,5 & 12,0 & 59,5 & 73,0 \\
\hline 80 & 9,4 & 10,0 & 4,4 & 5,0 & 5,0 & 5,0 & 11,4 & 12,0 & 64,0 & 73,0 \\
\hline 90 & 10,0 & 10,0 & 5,0 & 5,0 & 5,0 & 5,0 & 12,4 & 14,3 & 74,8 & 73,0 \\
\hline \multicolumn{11}{|c|}{2 anos de escolaridade $(n=32)$} \\
\hline \multicolumn{11}{|l|}{ Percentil } \\
\hline 2,5 & 5,0 & 6,0 & 2,0 & 2,0 & 2,0 & 3,0 & 0,0 & 1,0 & 0,0 & 9,0 \\
\hline 7,0 & 5,0 & 6,4 & 2,0 & 2,8 & 2,0 & 3,4 & 0,0 & 1,4 & 0,0 & 12,6 \\
\hline 10,0 & 5,4 & 7,0 & 2,4 & 4,0 & 2,4 & 4,0 & 0,0 & 2,0 & 0,0 & 18,0 \\
\hline 16,0 & 6,2 & 9,0 & 3,0 & 4,0 & 3,0 & 4,0 & 0,5 & 3,2 & 4,3 & 18,0 \\
\hline 20,0 & 6,8 & 9,0 & 3,0 & 4,0 & 3,0 & 4,0 & 1,6 & 4,0 & 14,4 & 18,0 \\
\hline 25,0 & 7,5 & 9,0 & 3,5 & 5,0 & 3,5 & 4,0 & 3,5 & 6,0 & 31,5 & 45,0 \\
\hline 30,0 & 8,0 & 9,0 & 4,0 & 5,0 & 4,0 & 5,0 & 5,2 & 7,0 & 45,0 & 45,0 \\
\hline 40,0 & 8,0 & 10,0 & 4,6 & 5,0 & 4,0 & 5,0 & 6,0 & 9,0 & 45,6 & 54,0 \\
\hline 50,0 & 9,0 & 10,0 & 5,0 & 5,0 & 4,0 & 5,0 & 7,0 & 10,0 & 46,0 & 55,0 \\
\hline 60,0 & 9,0 & 10,0 & 5,0 & 5,0 & 4,4 & 5,0 & 8,4 & 11,0 & 55,0 & 55,0 \\
\hline 70,0 & 9,8 & 10,0 & 5,0 & 5,0 & 5,0 & 5,0 & 9,0 & 12,0 & 55,0 & 73,0 \\
\hline 75,0 & 10,0 & 10,0 & 5,0 & 5,0 & 5,0 & 5,0 & 10,5 & 13,0 & 55,0 & 73,0 \\
\hline 80,0 & 10,0 & 10,0 & 5,0 & 5,0 & 5,0 & 5,0 & 12,6 & 13,0 & 58,6 & 73,0 \\
\hline 90,0 & 10,0 & 10,0 & 5,0 & 5,0 & 5,0 & 5,0 & 15,6 & 13,0 & 78,4 & 82,0 \\
\hline
\end{tabular}

Legenda: Pub = Escola Pública; Priv = Escola Privada.

Com exceção dos totais de interferências e reconstruções nos recontos (cuja hipótese é de menor ocorrência com aumento da escolaridade), observouse também que os estudantes de escolas particulares tiveram desempenho superior ao de crianças de escolas públicas em todos os escores analisados através do teste de Mann-Whitney: questionário total $(U=433,5$; $z=-2,72 ; p<0,01)$, questionário literal $(U=493,5$; $z=-2,15 ; p<0,05)$, questionário inferencial $(U=442,5$; $z=-2,78 ; p<0,01)$, total de cláusulas $(U=452 ; z=-2,42$; $p<0,05)$, porcentagem de cláusulas da cadeia principal da história $(U=468,5 ; z=-2,25 ; p<0,05)$ e total de inferências do reconto $(U=428 ; z=-2,75 ; p<0,05)$. O teste de Kruskal-Wallis seguido da prova de qui-quadrado mostra que houve diferenças significativas quanto ao desempenho no reconto $\left(\chi^{2}(1)=5,031, p<0,05\right)$, sendo que as crianças de escolas particulares tiveram seus recontos classificados nas categorias superiores. 


\section{História "O Coelho e o Cachorro" ( $4^{\circ}$ ano a $6^{\mathrm{a}}$ série do Ensino Fundamental)}

Os dados normativos para a tarefa de compreensão leitora - texto "O Coelho e o Cachorro" - são apresentados na Tabela 3, por anos completos de estudo e por tipo de escola. Conforme mostra a Tabela 3, foi verificado efeito teto no questionário (33,7\% da amostra pontuou em nível máximo no questionário total; $59,4 \%$ atingiu o máximo de acertos nas questões literais, e 42,6\% nas inferenciais), especialmente nas crianças de escola privada. Pode-se considerar que não foi encontrado efeito teto quanto ao reconto da história, já que somente $27,7 \%$ da amostra atinge a categoria máxima, o que representa um valor apenas ligeiramente maior do que o ponto de corte de $25 \%$ indicado por alguns autores. Não foi evidenciado efeito chão.

TABELA 3

Desempenho e percentis nas tarefas de compreensão de leitura da história "O Coelho e o Cachorro", por tipo de escola e anos completos de estudo.

\begin{tabular}{|c|c|c|c|c|c|c|c|c|c|c|}
\hline \multirow[b]{2}{*}{ Tipo de Escola } & \multicolumn{2}{|c|}{$\begin{array}{c}\text { Questionário } \\
\text { Total } \\
\text { (máximo }=10)\end{array}$} & \multicolumn{2}{|c|}{$\begin{array}{c}\text { Questionário } \\
\text { Literal } \\
\text { (máximo }=5)\end{array}$} & \multicolumn{2}{|c|}{$\begin{array}{l}\text { Questionário } \\
\text { Inferencial } \\
(\text { máximo }=5)\end{array}$} & \multicolumn{2}{|c|}{$\begin{array}{l}\text { Total Cláusulas } \\
\text { Recontadas } \\
\text { (máximo }=34 \text { ) }\end{array}$} & \multicolumn{2}{|c|}{$\begin{array}{c}\text { Porcentagem } \\
\text { Macroestrutura }\end{array}$} \\
\hline & $P u b$ & Priv & $P u b$ & Priv & $P u b$ & Priv & $P u b$ & Priv & $P u b$ & Priv \\
\hline \multicolumn{11}{|c|}{3 e 4 anos de escolaridade $(n=53)$} \\
\hline \multicolumn{11}{|l|}{ Percentil } \\
\hline 2,5 & 3,0 & 4,0 & 1,0 & 2,0 & 0,0 & 1,0 & 0,0 & 3,0 & 0,0 & 19,0 \\
\hline 7 & 3,3 & 5,0 & 1,7 & 2,5 & 0,3 & 1,0 & 0,7 & 9,1 & 4,0 & 40,9 \\
\hline 10 & 3,9 & 5,0 & 2,8 & 3,0 & 0,9 & 1,6 & 1,8 & 12,6 & 10,8 & 53,6 \\
\hline 16 & 4,0 & 5,8 & 3,0 & 3,0 & 2,0 & 2,0 & 3,0 & 13,0 & 19,0 & 60,6 \\
\hline 20 & 4,8 & 6,0 & 3,0 & 4,0 & 2,0 & 2,0 & 3,0 & 14,0 & 19,0 & 62,0 \\
\hline 25 & 5,0 & 6,0 & 3,0 & 4,0 & 2,0 & 2,0 & 3,8 & 15,0 & 23,5 & 62,0 \\
\hline 30 & 5,7 & 7,0 & 3,0 & 4,0 & 2,0 & 3,0 & 4,7 & 15,8 & 25,0 & 62,0 \\
\hline 40 & 6,0 & 8,0 & 4,0 & 4,4 & 2,6 & 4,0 & 8,2 & 16,4 & 37,0 & 75,0 \\
\hline 50 & 7,0 & 9,0 & 4,0 & 5,0 & 3,0 & 4,0 & 10,5 & 18,0 & 40,5 & 81,0 \\
\hline 60 & 8,0 & 9,0 & 4,0 & 5,0 & 3,0 & 5,0 & 12,8 & 19,0 & 52,4 & 81,0 \\
\hline 70 & 8,0 & 10,0 & 5,0 & 5,0 & 4,0 & 5,0 & 15,0 & 20,2 & 62,0 & 81,0 \\
\hline 75 & 8,3 & 10,0 & 5,0 & 5,0 & 4,0 & 5,0 & 15,0 & 21,0 & 63,5 & 87,0 \\
\hline 80 & 9,0 & 10,0 & 5,0 & 5,0 & 4,2 & 5,0 & 15,0 & 21,0 & 69,4 & 87,0 \\
\hline 90 & 10,0 & 10,0 & 5,0 & 5,0 & 5,0 & 5,0 & 16,6 & 22,8 & 76,2 & 93,4 \\
\hline \multicolumn{11}{|c|}{5 anos de escolaridade $(n=48)$} \\
\hline \multicolumn{11}{|l|}{ Percentil } \\
\hline 2,5 & 5,0 & 2,0 & 3,0 & 1,0 & 2,0 & 1,0 & 2,0 & 3,0 & 12,0 & 12,0 \\
\hline 7 & 6,0 & 3,6 & 3,0 & 1,5 & 2,0 & 1,5 & 3,0 & 5,7 & 18,7 & 22,3 \\
\hline 10 & 6,0 & 5,2 & 3,0 & 2,4 & 2,0 & 2,0 & 5,4 & 8,2 & 19,0 & 32,2 \\
\hline 16 & 6,5 & 6,5 & 4,0 & 4,0 & 2,5 & 2,5 & 6,5 & 11,1 & 25,0 & 46,9 \\
\hline 20 & 7,0 & 7,4 & 4,0 & 4,0 & 3,0 & 3,0 & 7,6 & 13,4 & 36,4 & 58,4 \\
\hline 25 & 7,0 & 8,5 & 4,0 & 4,0 & 3,0 & 3,5 & 9,0 & 14,5 & 50,0 & 65,5 \\
\hline 30 & 7,4 & 9,0 & 4,0 & 4,6 & 3,0 & 4,0 & 10,0 & 15,6 & 52,4 & 69,0 \\
\hline 40 & 9,0 & 9,0 & 5,0 & 5,0 & 4,0 & 4,0 & 14,0 & 17,0 & 57,2 & 75,0 \\
\hline 50 & 9,0 & 9,0 & 5,0 & 5,0 & 4,0 & 5,0 & 15,0 & 18,0 & 69,0 & 81,0 \\
\hline 60 & 9,8 & 9,2 & 5,0 & 5,0 & 5,0 & 5,0 & 16,0 & 19,2 & 75,0 & 87,0 \\
\hline 70 & 10,0 & 10,0 & 5,0 & 5,0 & 5,0 & 5,0 & 18,2 & 20,4 & 81,0 & 87,0 \\
\hline 75 & 10,0 & 10,0 & 5,0 & 5,0 & 5,0 & 5,0 & 21,0 & 21,0 & 81,0 & 87,0 \\
\hline 80 & 10,0 & 10,0 & 5,0 & 5,0 & 5,0 & 5,0 & 21,4 & 21,6 & 83,4 & 91,2 \\
\hline 90 & 10,0 & 10,0 & 5,0 & 5,0 & 5,0 & 5,0 & 25,0 & 23,8 & 87,0 & 94,0 \\
\hline
\end{tabular}

Legenda: Pub = Escola Pública; Priv = Escola Privada. 
O teste Mann-Whitney mostrou diferenças significativas de desempenho entre os grupos conforme o tempo de escolarização, sendo que as crianças com cinco anos de estudo apresentaram desempenho superior ao das de quatro anos em: questionário total ( $U=968 ; z=-2,12 ; p<0,05)$, questionário inferencial $(U=1025,5 ; z=-1,9 ; p<0,05)$ e total de inferências do reconto $(U=865,5 ; z=-2,9 ; p<0,01)$. Quanto ao reconto da história, observou-se que os estudantes de escolas particulares tiveram desempenho superior ao de crianças de escolas públicas nos escores: total de cláusulas recontadas $(U=729 ; z=-3,63 ; p<0,01)$, porcentagem de cláusulas da cadeia principal da história ( $U=701 ; z=-3,84 ; p<0,01)$, e total de inferências do reconto $(U=948,5 ; z=-2,23 ; p<0,05)$. Como todas essas variáveis quantitativas compõem os critérios para a classificação dos recontos nas diferentes categorias, já era esperado o resultado do teste de Kruskal-Wallis seguido da prova de qui-quadrado, que aponta para diferenças significativas nas médias dos ranques das categorias de recontos dos grupos de escolas diferentes $\left(\chi^{2}(1)=12,092, p<0,01\right)$. As crianças de escolas particulares obtiveram desempenho significativamente superior que as de escolas públicas, tendo seus recontos classificados nas categorias mais elevadas.

\section{DISCUSSÃO}

Observa-se que, tanto nas medidas baseadas na história "O Coelho e o Cachorro", como nas tarefas realizadas a partir da história "A Coisa", houve diferenças de desempenho por anos completos de estudo formal, na escola privada e na pública. Espera-se que as crianças progridam no desempenho em compreensão leitora ao longo das séries/anos escolares e com o aumento da idade. Nas séries/anos iniciais, o progresso na habilidade de reconhecimento de palavras tem uma participação importante no desenvolvimento da própria compreensão, uma vez que se torna mais automática e precisa (Seigneuric \& Ehrlich, 2005). Acurácia e fluência no reconhecimento de palavras são aspectos dos quais a compreensão leitora propriamente dita depende (Cain, Oakhill, \& Bryant, 2004). Com o avanço da escolaridade diminui o peso do reconhecimento da palavra na habilidade de compreensão leitora, e os progressos na habilidade passam a depender também de fatores relacionados ao desenvolvimento cognitivo e linguístico da criança (Bowey, 2000; Kendeou, Muis, \& Fulton, 2010). Assim, é também compreensível a diferença significativa de desempenho verificada entre o grupo composto de crianças de três e quatro anos de estudo e aquele composto por crianças com 5 anos de estudo. A realização de inferências, por exemplo, evolui com a idade/escolaridade (Dawson \& Guare, 2010).

As diferenças de desempenho por tempo de escolaridade aparecem na tarefa de questionário, especificamente nas questões literais, para a amostra que realizou a leitura de "A Coisa" e nas questões inferenciais para a amostra que leu "O Coelho e o Cachorro". Pode-se hipotetizar que nas questões mais difíceis e sensíveis para verificar a compreensão mais profunda do texto - as questões inferenciais as diferenças começam a aparecer nas séries mais adiantadas. Mas também é preciso levar em conta que o fato de serem textos e questões diferentes usados nas duas amostras pode explicar, pelo menos em parte, as diferenças de desempenho entre elas.

Não houve diferenças de desempenho quanto ao reconto (em nenhuma das variáveis que o avaliam) em relação à escolaridade das crianças. Restaria tentar explicar porque aparece um efeito de série sobre o desempenho no questionário, e não sobre o reconto. Há que se considerarem aqui as diferenças nas duas tarefas propostas, pois diferentes tarefas de compreensão leitora podem corresponder a diferentes mecanismos cognitivos e linguísticos. O reconto (evocação livre) envolve recursos de memória verbal - recordação livre, produção verbal, capacidade de selecionar os elementos mais importantes e de inferir fatos implícitos (Brandão \& Spinillo, 1998; McVay \& Kane, 2012). As questões sobre o texto envolvem, sobretudo, o reconhecimento da informação, neste sentido oferecendo menos demanda em termos de memória, mas, por outro lado, no que diz respeito às questões inferenciais, exigem um raciocínio sobre a informação do texto (Goldmann \& Wolfe, 2001). O raciocínio inferencial exigido por parte das questões pode ter se constituído em uma dificuldade maior às crianças de quarta série - de fato foi precisamente quanto às questões inferenciais que apareceu a diferença entre a quarta e a sexta séries no grupo que leu "O Coelho e o Cachorro". Confirmando a capacidade de realizar inferências como uma habilidade que diferencia os grupos a partir da quarta série, o número de inferências realizadas no reconto apresentou diferenças significativas entre a quarta e a sexta séries.

Os resultados deste estudo evidenciam diferenças de desempenho nos recontos das histórias em relação ao tipo de escola que a criança frequenta. Diferenças de desempenho em leitura, avaliações cognitivas e desempenho escolar em relação ao tipo de escola têm sido encontradas por outros autores (Casarin, Wong, Parente, Salles, \& Fonseca, 2012; Castillo et al., 2011; Phillips \& Lonigan, 2010; Villaseñor, Martín, Díaz, Rosselli, \& Ardila, 2009). Provavelmente, a variável 
tipo de escola está relacionada a outros fatores que determinam a qualidade do ambiente em que a criança vive, como o nível socioeconômico, a oferta de materiais e atividades de lazer, o método de ensino da escola, a quantidade e qualidade de estimulação e da relação que a criança possui com pais, colegas e professores (Engel, Santos, \& Gathercole, 2008; Gardinal \& Marturano, 2007; Piccolo et al., 2012). Todos esses aspectos caracterizam um ambiente mais ou menos facilitador para o desenvolvimento da linguagem como um todo, incluindo a compreensão textual (Phillips \& Lonigan, 2010).

Por fim, os diferentes desempenhos conforme escolaridade e tipo de escola sugerem evidências de validade de critério para o instrumento. Observam-se valores diferentes em todas as faixas de escolaridade (para ambos os tipos de escola) no percentil sete (equivalente ao escore $\mathrm{z}$ de -1,5 DP, sugestivo de déficit (Strauss, Sherman, Spreen, \& Slick, 2006). Quanto a efeitos teto e chão, o fato de ter sido verificado efeito teto no questionário mostra que esta tarefa se mostrou menos sensível para diferenças de desempenho do que $\mathrm{o}$ reconto. $\mathrm{O}$ fato de não ter sido evidenciado efeito chão em nenhuma tarefa mostra que o instrumento é potencialmente sensível aos casos de dificuldade em compreensão leitora.

\section{CONSIDERAÇÕES FINAIS}

O instrumento apresentado é de fácil aplicação, embora exija um espaço reservado para entrevista individual com o examinando. Além da folha de texto e da folha com as questões, o material necessário envolve apenas um gravador de voz. Alguma complexidade está presente na correção dos protocolos de reconto, o que envolve até certo ponto uma avaliação qualitativa. Nem sempre é simples decidir se a cláusula está ou não presente no reconto da criança, assim como não é simples a identificação de inferências, interferências e reconstruções. Tal complexidade, entretanto, não inviabiliza o uso do reconto (evocação livre) na avaliação da compreensão leitora, e, pelo contrário, enriquece a avaliação desta habilidade que, de resto, é ela própria muito complexa.

$\mathrm{O}$ instrumento pode ser usado tanto em um espaço clínico, de pesquisa, quanto no espaço escolar, sendo, neste último contexto, próprio para detecção de casos de dificuldade em compreensão leitora. Além disso, a ferramenta permite bases para intervenção. Recomenda-se que seja aliada à avaliação dos demais componentes de leitura: reconhecimento palavra, fluência, assim como das demais funções neuropsicológicas relacionadas à compreensão leitora. Salienta-se que, especialmente nas avaliações de habilidades verbais, é importante considerar os contextos em que a avaliação foi realizada, características das crianças e do ambiente onde vivem e outras particularidades da testagem que podem influenciar o desempenho do testando. Além disso, é importante que a habilidade de compreensão de leitura de textos narrativos, como é feita pelo presente instrumento, seja avaliada também através da leitura de textos expositivos.

\section{REFERÊNCIAS}

Angelini, A. L., Alves, I. C. B., Custódio, E. M., Duarte, W. F., \& Duarte, J. L. M. (1999). Matrizes progressivas coloridas de Raven: escala especial [Manual]. São Paulo: Centro Editor de Testes e Pesquisas em Psicologia.

Associação Brasileira de Empresas de Pesquisa [ABEP] (2009). Critério de Classificação Econômica Brasil. Recuperado em 30 setembro, 2013, de http://www.abep.org/novo/Utils/FileGenerate.ashx?id=197

Bowey, J. A. (2000). Recent developments in language acquisition and reading research: the phonological basis of children's reading difficulties. Australian Educational and Developmental Psychologist, 17(1), 5-31.

Brandão, A. C. P. \& Spinillo, A. G. (1998). Aspectos gerais e específicos na compreensão de textos. Psicologia: Reflexão e Crítica, 11(2), 253-272. http://dx.doi.org/10.1590/S0102-79721998000200006

Brandão, A. C. P. \& Spinillo, A. G. (2001). Produção e compreensão de textos em uma perspectiva de desenvolvimento. Estudos de Psicologia, 6(1), 51-62. http://dx.doi.org/10.1590/S1413-294X2001000100006

Brito, G. N. (1987). The Conners abbreviated teacher rating scale: development of norms in Brazil. Journal of Abnormal Child Psychology, 15, 511-518. http://dx.doi.org/10.1007/BF00917237

Cain, K., Oakhill, J., \& Bryant, P. (2004). Children's reading comprehension ability: concurrent prediction by working memory, verbal ability, and component skills. Journal of Educational Psychology, 96(1), 31-42. http://dx.doi. org/10.1037/0022-0663.96.1.31

Casarin, F. S., Wong, C. E. I., Parente, M. A. M. P., Salles, J. F., \& Fonseca, R. P. (2012). Comparação de desempenho neuropsicológico entre estudantes de escolas públicas e privadas brasileiras. Spanish Journal of Psychholog, 15(3), 942-951. http://dx.doi.org/10.5209/rev_SJOP.2012.v15.n3.39386

Castillo, R., Ruiz, J. R., Chillón, P., Jiménez-Pavón, D., Esperanza-Díaz, L., Moreno, L. A., \& Ortega, F. B. (2011). Associations between parental educational/occupational levels and cognitive performance in Spanish adolescents: the AVENA study. Psicothema, 23(3), 349-355. 
Corso, H. V. \& Salles, J. F. (2009). Relação entre leitura de palavras isoladas e compreensão de leitura textual em crianças. Letras de Hoje, 44(3) 28-35.

Corso, H. V., Sperb, T. M., \& Salles, J. F. (2012). Desenvolvimento de instrumento de compreensão leitora a partir de reconto e questionário. Neuropsicologia Latinoamericana, 4(1), 22-28.

Corso, H. V., Sperb, T. M., \& Salles, J. F. (no prelo). Compreensão leitora: modelos de processamento e relações com outras habilidades cognitivas. In A. Roazzi, F. R. R. Justi, \& J. F. Salles (Org.). A aprendizagem da leitura e da escrita: Contribuições de pesquisas.

Cuetos, F., Rodrigues, B., \& Ruano, E. (1998). Provas de avaliação dos processos de leitura - PROLEC [Manual]. (S. A. Capellini, A. M. Oliveira, \& F. Cuetos, adaptação brasileira). São Paulo: Casa do Psicólogo.

Dawson, P. \& Guare, R. (2010). Executive skills in children and adolescents (2nd ed). New York: Guilford Press.

Engel, P. M. J., Santos, F. H., \& Gathercole, S. E. (2008). Are working memory measures free of socioeconomic influence? Journal of Speech, Language, and Hearing Research, 51, 1580-1587. http://dx.doi.org/10.1044/10924388(2008/07-0210)

Ehri, L. C. (2010). Development of sight word reading: phases and findings. In M. J. Snowling, \& C. Hulme (Eds.). The science of reading: A handbook (4th ed.) (pp. 135-154). Oxford, UK: Blackwell.

Gardinal, E. C. \& Marturano, E. M. (2007). Meninos e meninas na Educação Infantil: associação entre comportamento e desempenho. Psicologia em Estudo, 12, 541-551. http://dx.doi.org/10.1590/S1413-73722007000300011

Goldmann, S. R. \& Wolfe, M. B. W. (2001). Text comprehension: Models in psychology. International Encyclopedia of the Social \& Behavioral Sciences, 15625-15631. http://dx.doi.org/10.1016/B0-08-043076-7/01542-4

Hulme, C. \& Snowling, M. J. (2011). Children's reading comprehension difficulties: nature, causes, and treatments. Current Directions in Psychological Science, 20(3), 139-142. http://dx.doi.org/10.1177/0963721411408673

Johnston, A. M., Barnes, M., \& Desrochers, A. (2008). Reading comprehension: developmental processes, individual differences, and interventions. Canadian Psychology, 49(2), 125-132. http://dx.doi.org/10.1037/0708-5591.49.2.125

Kendeou, P., Muis, K. R., \& Fulton, S. (2010). Reader and text factors in reading comprehension processes. Journal of Research in Reading, 34(4), 365-383. http://dx.doi.org/10.1111/j.1467-9817.2010.01436.x

Kintsch, W. (1988). The role of knowledge in discourse comprehension: a construction-integration model. Psychological Review, 95(2), 163-182. http://dx.doi.org/10.1037/0033-295X.95.2.163

Kintsch, W. (1998). Comprehension: A paradigm for cognition. New York: Cambridge University Press.

Marotto, C. M. (2000). Introducción a los modelos cognitivos de la comprensión del lenguage. Universidad de Buenos Aires: Editorial Universitária de Buenos Aires.

McHorney, C. A., Ware, J. E., Lu J. F. R., \& Sherbourne, C. D. (1994). The MOS 36-item short-form health survey (SF-36): III. Tests of data quality, scaling assumptions and reliability across diverse patient groups. Medical Care, 32, 40-66. http://dx.doi.org/10.1097/00005650-199401000-00004

McVay, J. C. \& Kane, M. J. (2012). Why does working memory capacity predict variation in reading comprehension? On the influence of mind wandering and executive attention. Journal of Experimental Psychology: General, 141(2), 302-320. http://dx.doi.org/10.1037/a0025250

Oakhill, J. \& Cain, K. (2006). Reading processes in reading. In K. Brown (Ed.), Encyclopedia of Language \& Linguistics (2nd ed.) (pp. 379-386). Oxford: Elsevier.

Ouellette, G. \& Beers, A. (2010). A not-so-simple view of reading: how oral vocabulary and visual-word recognition complicate the story. Reading and Writing, 23(2), 189-208. http://dx.doi.org/10.1007/s11145-008-9159-1

Parente, M. A., Saboskinski, A. P., Ferreira, E., \& Nespoulous, J. (1999). Memória e compreensão da linguagem no envelhecimento. Estudos Interdisciplinares sobre o Envelhecimento (v. 1, pp. 57-76). Porto Alegre: PROREXT/UFRGS.

Phillips, B. M. \& Lonigan, C. J. (2010). Social correlates of Emergent Literacy. In M. J. Snowling, \& C. Hulme (Eds.). The science of reading: A handbook (4th ed.) (pp. 173-187). Oxford, UK: Blackwell.

Piccolo, L. R. \& Salles, J. F. (2013). Vocabulário e memória de trabalho predizem desempenho em leitura de crianças. Revista Psicologia: Teoria e Prática, 15(2), 180-191.

Piccolo, L. R., Falceto, O. G., Fernandes, C. L., Levandowski, D. C., Grassi-Oliveira, R., \& Salles, J. F. (2012). Variáveis psicossociais e desempenho em leitura de crianças de baixo nível socioeconômico. Psicologia: Teoria e Pesquisa, 28(4), 389-398. http://dx.doi.org/10.1590/S0102-37722012000400004

Prata, M. (2005). O coelho e o cachorro. In M. A. Negrinho. Aulas de Redação - $\sigma^{a}$ série (pp. 42-43). São Paulo: Ática.

Raven, J. C., Court, J. H., \& Raven, J. (1986). Coloured progressive matrices. London: H. K. Lewis.

Rocha, R. (1997). A Coisa. In: C. S. Carvalho \& M. D. Baraldi (Eds.), Construindo a escrita: leitura e interpretação de textos (pp. 86-95). São Paulo: África.

Salles, J. F. \& Parente, M. A. M. P. (2004). Compreensão textual em alunos de segunda e terceira séries: uma abordagem cognitiva. Estudos de Psicologia, 9(1), 71-80. http://dx.doi.org/10.1590/S1413-294X2004000100009

Saraiva, R., Moojen, S., \& Munarski, R. (2009). Avaliação da compreensão leitora de textos expositivos para fonoaudiólogos e psicopedagogos. São Paulo: Casa do Psicólogo.

Schmitt, N., Jiang, X., \& Grabe, W. (2011). The percentage of words known in a text and reading comprehension. The Modern Language Journal, 95(1), 26-43. http://dx.doi.org/10.1111/j.1540-4781.2011.01146.x 
Seigneuric, A. \& Ehrlich, M. (2005). Contribution of working memory capacity to children's reading comprehension: a longitudinal investigation. Reading and Writing, 18, 617-656. http://dx.doi.org/10.1007/s11145-005-2038-0

Siqueira, M. \& Lamprecht, R. R. (2007). As metáforas primárias na aquisição da linguagem: um estudo interlinguístico. D.E.L.T.A., 23(2), 245-272. http://dx.doi.org/10.1590/S0102-44502007000200004

Sotelo-Dynega, M., Flanagan, D. P., \& Alfonso, V. C. (2011). One Overview of Specific Learning Disabilities. In D. P. Flanagan \& V. C. Alfonso (Eds.), Essentials of specific learning disability identification (pp. 1-19). New Jersey, NJ: Wiley.

Trabasso, T., Van Den Broek, P., \& Suh, S. (1989). Logical necessity and transitivity of causal relations in the representation of stories. Discourse Processes, 12, 1-25. http://dx.doi.org/10.1080/01638538909544717

van Kraayenoord, C. E., Beinicke, A., Schlagmuller, M., \& Schneider, W. (2012). Word identification, metacognitive knowledge, motivation and reading comprehension: An Australian study of Grade 3 and 4 pupils. Australian Journal of Language and Literacy, 35(1), 51-68.

Villaseñor, E. M., Sanz Martín, A., Gumá Díaz, E., Ardila, A., Rosselli, M., \& Ardila, A. (2009). Influencia del nivel educativo de los padres, el tipo de escuela y el sexo en el desarrollo de la atención y la memoria: effects of parents' educational level, school type and gender on the development of attention and memory. Revista Latinoamericana de Psicología, 41(2), 257-276.

\footnotetext{
Autores:

Helena Vellinho Corso - Doutora, Universidade Federal do Rio Grande do Sul.

LuCIANE Rosa PICCOLO - Doutora, Universidade Federal do Rio Grande do Sul.

CAmila Schorr Miná - Psicóloga, Universidade Federal do Rio Grande do Sul.

JeRusa Fumagalli SALleS - Doutora, Universidade Federal do Rio Grande do Sul.
}

Endereço para correspondência:

Helena Vellinho Corso

E-mail: hvcorso@gmail.com

Recebido em: 07.04.14

Aceito em: 13.10 .14 